\title{
SYMMETRY PROPERTIES OF A GENERALIZED KORTEWEG-DE VRIES EQUATION AND SOME EXPLICIT SOLUTIONS
}

\author{
PAUL BRACKEN \\ Received 18 October 2004
}

The symmetry group method is applied to a generalized Korteweg-de Vries equation and several classes of group invariant solutions for it are obtained by means of this technique. Polynomial, trigonometric, and elliptic function solutions can be calculated. It is shown that this generalized equation can be reduced to a first-order equation under a particular second-order differential constraint which resembles a Schrödinger equation. For a particular instance in which the constraint is satisfied, the generalized equation is reduced to a quadrature. A condition which ensures that the reciprocal of a solution is also a solution is given, and a first integral to this constraint is found.

\section{Introduction}

Recently, there has been interest in the fact that a particular generalization of the classical korteweg-de Vries (KdV) equation can support a new type of solitary wave, which has been referred to as a compacton in the literature. This type of wave has a compact support and a width which is independent of the amplitude of the wave $[11,12]$. This equation is defined by the real parameters $(m, n)$ and given in terms of the function $u(x, t)$ by the fully nonlinear KdV equation [4]

$$
u_{t}+\left(u^{m}\right)_{x}+\left(u^{n}\right)_{x x x}=0
$$

The classical KdV equation has been studied extensively $[1,3,6]$, in particular, by means of the inverse scattering method, and the Bäcklund transformation has been determined [7], but relatively few papers which mention (1.1) have appeared.

Dynamical solitons appear as a result of a balance between weak nonlinearity and dispersion. However, it has been shown numerically at least, that when the wave dispersion is purely nonlinear, some novel features in the nonlinear dynamics may be observed. The most striking and novel is the existence of a new type of soliton, which has been referred to as a compacton in certain quarters [4]. This is in contrast to the standard KdV soliton solution, which narrows as the amplitude increases. The width of this new type of soliton is independent of the amplitude. 
The fully nonlinear KdV equation is of some importance to study on physical grounds [5]. For example, several equations pertaining to a discrete lattice have continuous limits which are partial differential equations either resembling (1.1) or having compacton properties similar to those of the generalized $\mathrm{KdV}$ equation. As an example, consider a one-dimensional lattice in which each atom interacts only with its nearest neighbors by purely anharmonic forces. If $x_{n}(t)$ is the dimensionless displacement of the $n$th atom from its equilibrium position, and the atoms interact via quartic anharmonic potentials, the equation of motion for the $n$th atom is given by

$$
\frac{d^{2} x_{n}}{d t^{2}}=\left[\left(x_{n+1}-x_{n}\right)^{3}+\left(x_{n-1}-x_{n}\right)^{3}\right]
$$

where dimensionless units have been used. In the long- and short-wavelength limits, the resulting partial differential equations have properties similar to the generalized $\mathrm{KdV}$ equation (1.1).

Not many solutions to (1.1) are known at the moment, and so it would be useful to have whatever new results that can be obtained at this point. In this paper, it will be shown that the symmetry group of (1.1) can be determined. By this, we mean the classical symmetry group $[8,9,10]$ of $(1.1)$. In this procedure, the coefficients of the infinitesimal generator $\mathbf{v}$ of a hypothetical one-parameter symmetry group are unknown functions of $t, x$, and $u$. The coefficients of the prolonged infinitesimal generator $p r^{(3)} \mathbf{v}$ will be explicit expressions involving the partial derivatives of the coefficient functions with respect to both $t, x$, and $u$. Eliminating any dependencies among the derivatives of $u$ modulo the original system, the coefficients of the remaining unconstrained partial derivatives of $u$ are equated to zero. This results in a large number of determining equations for the symmetry group, which are solved. The symmetry group will first be determined for the case $n=1$ in (1.1). The Lie symmetry algebra is found to be a subalgebra of the usual Lie algebra for the standard KdV equation, which corresponds to the case $m=2$ here. When $m=1$, the equation becomes completely linear in $u$, and when $m=2$, the standard KdV symmetries are found. The corresponding symmetry variables can be calculated, and it is found that symmetry reduction to ordinary differential equations can be carried out. The calculation has been repeated for the case in which $n$ is not one, and the symmetry group is found to be much more restrictive in this case. The vector fields which are obtained simply correspond to translations in space and translations in time. Nonetheless, it is shown how some of these reductions can be used to integrate (1.1) and to produce explicit examples of solutions to (1.1). Finally, a connection is made between (1.1) when $n=1$ and a nonlinear type of Schrödinger equation, which acts as a differential constraint. This result can also be used to generate new solutions to (1.1). It is shown how this can be done by obtaining a quadrature for the solution in terms of a symmetry variable.

\section{Analysis of the equation with one power of $u$ in the third derivative}

Consider the family of fully nonlinear $\mathrm{KdV}$ equations given by (1.1) and written in the equivalent form with $n=1$ and $m \neq 0$ as

$$
u_{t}+u_{x x x}+m u^{m-1} u_{x}=0
$$


Three conservation laws for (2.1) exist and are given by

$$
\begin{gathered}
u_{t}+\left(u^{m}+u_{x x}\right)_{x}=0, \quad\left(u^{2}\right)_{t}+\left(\frac{2 m}{m+1} u^{m+1}+2 u u_{x x}-u_{x}^{2}\right)_{x}=0 \\
\left(\frac{1}{m+1} u^{m+1}-\frac{1}{2} u_{x}^{2}\right)_{t}+\left(u^{m} u_{x x}+\frac{1}{2} u_{x x}^{2}+\frac{1}{2} u^{2 m}-u_{x} u_{x x x}-m u^{m-1} u_{x}^{2}\right)_{x}=0
\end{gathered}
$$

Of course, an equation of the form (1.1) is trivially a conservation law itself. Let

$$
\mathbf{v}=\xi(x, t, u) \frac{\partial}{\partial x}+\tau(x, t, u) \frac{\partial}{\partial t}+\varphi(x, t, u) \frac{\partial}{\partial u}
$$

be a vector field on $X \times U$. All possible coefficient functions $\xi$, $\tau$, and $\varphi$ are to be determined such that the corresponding one-parameter group $\exp (\epsilon \mathbf{v})$ is a symmetry group of this equation. To obtain the prolonged equation, the operator

$$
p^{(3)} \mathbf{v}=\mathbf{v}+\varphi^{x} \frac{\partial}{\partial u_{x}}+\varphi^{t} \frac{\partial}{\partial u_{t}}+\varphi^{x x} \frac{\partial}{\partial u_{x x}}+\varphi^{x t} \frac{\partial}{\partial u_{x t}}+\varphi^{t t} \frac{\partial}{\partial u_{t t}}+\varphi^{x x x} \frac{\partial}{\partial u_{x x x}}
$$

is applied to (2.1). Only the terms relevant to this case have been retained in writing (2.4). Applying (2.4) to (2.1), it is found that the coefficient functions in $p r^{(3)} \mathbf{v}$ satisfy the equation

$$
\varphi^{t}+\varphi^{x x x}+m \varphi^{x} u^{m-1}+m(m-1) \varphi u^{m-2} u_{x}=0 .
$$

The coefficient functions in (2.4) can be calculated and then substituted into (2.5) to obtain

$$
\begin{aligned}
\varphi_{t}- & \xi_{t} u_{x}+\left(\varphi_{u}-\tau_{t}\right) u_{t}-\xi_{u} u_{x} u_{t}-\tau_{u} u_{t}^{2}+D_{x}^{3} \varphi-u_{x} D_{x}^{3} \xi-u_{t} D_{x}^{3} \tau-3 u_{x x} D_{x}^{2} \xi-3 u_{x t} D_{x}^{2} \tau \\
& -3 u_{x x x} D_{x} \xi-3 u_{x x t} D_{x} \tau+m \varphi_{x} u^{m-1}+m\left(\varphi_{u}-\xi_{x}\right) u^{m-1} u_{x}-m \tau_{x} u^{m-1} u_{t} \\
& -m \xi_{u} u^{m-1} u_{x}^{2}-m \tau_{u} u^{m-1} u_{x} u_{t}+m(m-1) \varphi u^{m-2} u_{x}=0 .
\end{aligned}
$$

In (2.6), $D_{w}$ represents the total derivative of the indicated function with respect to $w$. Substituting the total derivatives into (2.6) and replacing the term $u_{t}$ using (2.1), the next step is to collect all like derivative terms together and equate the coefficients to zero to obtain the set of determining equations. The highest derivative terms $u_{x x t}$ and $u_{x} u_{x x t}$ provide the constraints $\tau_{x}=0$ and $\tau_{u}=0$, respectively. These imply that $\tau=\tau(t)$ is a function of only the $t$ variable. The coefficient of $u_{x x}^{2}$ gives the condition $\xi_{u}=0$, hence $\xi$ is independent of $u$.

The terms which multiply $u_{x x x}$ give the constraint

$$
\tau_{t}=3 \xi_{x}
$$

Since $\tau$ depends only on $t$, this equation can be integrated to obtain $\xi$ as a linear function of $x$ :

$$
\xi(x, t)=\frac{1}{3} \tau_{t}(t) x+\sigma(t) .
$$


The coefficients of $u_{x} u_{x x}$ and $u_{x x}$ give the constraints $\varphi_{u u}=0$ and $\varphi_{x u}=\xi_{x x}$. Since $\xi(x, t)$ is just linear in $x$, this pair of equations reduces to simply $\varphi_{u u}=0$ and $\varphi_{x u}=0$. These constraints imply that $\varphi$ is at most linear in the function $u$, and the coefficient of $u$ is a function of $t$ alone. The remaining terms of (2.6) under these constraints are given by

$$
\begin{gathered}
\varphi_{t}-\xi_{t} u_{x}-m\left(\varphi_{u}-\tau_{t}\right) u^{m-1} u_{x}+m \varphi_{x} u^{m-1}+m\left(\varphi_{u}-\xi_{x}\right) u^{m-1} u_{x} \\
+m(m-1) \varphi u^{m-2} u_{x}+\varphi_{x x x}=0
\end{gathered}
$$

The term which multiplies $u_{x}$ is given by

$$
-\xi_{t}-m\left(\varphi_{u}-\tau_{t}\right) u^{m-1}+m\left(\varphi_{u}-\xi_{x}\right) u^{m-1}+m(m-1) u^{m-2} \varphi=0,
$$

and the remaining term requires that

$$
\varphi_{t}+m u^{m-1} \varphi_{x}+\varphi_{x x x}=0
$$

Collecting like terms in (2.10), we can write

$$
-\xi_{t}+m\left(\tau_{t}-\xi_{x}\right) u^{m-1}+m(m-1) \varphi u^{m-2}=0 .
$$

Substituting (2.8) and $\varphi(t, u)=\alpha(t) u+\beta$ into (2.12), we obtain that

$$
-\frac{1}{3} \tau_{t t}(t) x-\sigma_{t}(t)+m\left(\frac{2}{3} \tau_{t}+(m-1) \alpha\right) u^{m-1}+m(m-1) \beta u^{m-2}=0 .
$$

In order that the coefficient of $x$ vanishes, we must have $\tau_{t t}(t)=0$, hence $\tau(t)=c_{2}+c_{4} t$. The coefficients of the remaining powers of $u$ must also be equated to zero, and how this is carried out depends on the value of $m$ to some extent. There are three cases to consider, and we discuss each of these cases in turn.

(i) Suppose that $m \neq 1,2$, then there are three independent terms in (2.13), which yield the constraints

$$
\sigma_{t}(t)=0, \quad \frac{2}{3} \tau_{t}+(m-1) \alpha=0, \quad \beta=0 .
$$

The general solution to (2.17) is given by

$$
\sigma=c_{1}, \quad \alpha=-\frac{2}{3(m-1)} c_{4}, \quad \beta=0 .
$$

Therefore, the components of the vector field can be written as

$$
\xi=\frac{1}{3} c_{4} x+c_{1}, \quad \varphi=\frac{2 c_{4}}{3(m-1)} u, \quad \tau=c_{2}+c_{4} t,
$$

and the vector field (2.3) can be written as

$$
\mathbf{v}=\left(\frac{1}{3} c_{4} x+c_{1}\right) \frac{\partial}{\partial x}+\left(c_{2}+c_{4} t\right) \frac{\partial}{\partial t}-\frac{2 c_{4}}{3(m-1)} u \frac{\partial}{\partial u} .
$$


(ii) When $m=0$ or $m=1,(2.1)$ reduces to a linear equation. When $m=1$, the last term in (2.13) is absent, giving

$$
\sigma_{t}(t)=0, \quad \tau_{t}(t)=0 .
$$

These imply that

$$
\sigma=c_{1}, \quad \tau=c_{2}, \quad \varphi(t, u)=\alpha(t) u+\beta .
$$

Substituting $\varphi(t, u)$ into (2.11), it is found that $\alpha$ and $\beta$ must be constants and the vector field is given by

$$
\mathbf{v}=c_{1} \frac{\partial}{\partial x}+c_{2} \frac{\partial}{\partial t}+(a u+b) \frac{\partial}{\partial u}
$$

with $a, b$ decoupled from $c_{1}$ and $c_{2}$.

When $m=0,(2.1)$ is a third-order linear equation and (2.13) implies that $\tau_{t t}(t)=0$ and $\sigma_{t}(t)=0$. Therefore, $\tau(t)=c_{2}+c_{4} t$ and $\sigma=c_{1}$.

(iii) Finally, when $m=2$, the last term in (2.13) can be grouped with the first term to give the pair of equations

$$
\frac{2}{3} \tau_{t}(t)+\alpha=0, \quad \sigma_{t}(t)=2 \beta
$$

Integrating these, we find that

$$
\xi=\frac{1}{3} c_{4} x+2 c_{3} t+c_{1}, \quad \tau=c_{2}+c_{4} t, \quad \varphi=-\frac{2}{3} c_{4} u+c_{3},
$$

and the vector field (2.3) is given explicitly as follows:

$$
\mathbf{v}=\left(\frac{1}{3} c_{4} x+2 c_{3} t+c_{1}\right) \frac{\partial}{\partial x}+\left(c_{2}+c_{4} t\right) \frac{\partial}{\partial t}+\left(c_{3}-\frac{2}{3} c_{4} u\right) \frac{\partial}{\partial u}
$$

The case $m=2$ of course corresponds exactly to the classical nonlinear KdV equation. It can be seen then that for the general case $m \neq 1$, the symmetry generators are very close in structure to the classical KdV case. In fact, there are three independent generators, or vector fields specified by (2.17), which are shown in Table 2.1. These generate a Lie subalgebra of the algebra for the classical KdV equation. Exponentiation shows that if $u=$ $f(x, t)$ is a solution of (2.1), then so are the functions in the second column of Table 2.1.

A symmetry reduction can be carried out using $\mathbf{v}_{\mathbf{3}}$ for case (i). This gives rise to the following system:

$$
\frac{d x}{x}=\frac{d t}{3 t}=-\frac{1}{2}(m-1) \frac{d u}{u} .
$$

Integrating the first pair provides the symmetry variable defined by

$$
\chi=t^{-1 / 3} x
$$


Table 2.1. Symmetry algebra spanning vector fields and exponentiated solutions for (2.1). The case $m=2$ corresponds to the usual $\mathrm{KdV}$ equation.

\begin{tabular}{l|cc}
\hline Case & Symmetry vector field & Exponentiated solution \\
\hline$m \neq 1,2$ & $\mathbf{v}_{1}=\frac{\partial}{\partial x}$ & $u^{(1)}=f(x-\epsilon, t)$ \\
$\mathbf{v}_{2}=\frac{\partial}{\partial t}$ & $u^{(2)}=f(x, t-\epsilon)$ \\
\hline$m=1$ & $\mathbf{v}_{3}=x \frac{\partial}{\partial x}+3 t \frac{\partial}{\partial t}-\frac{2}{m-1} u \frac{\partial}{\partial u}$ & $u^{(3)}=f\left(e^{-\epsilon} x, e^{-3 \epsilon} t\right) e^{-2 \epsilon /(m-1)}$ \\
\hline $\mathbf{v}_{1}=\frac{\partial}{\partial x}$ & $u^{(1)}=f(x-\epsilon, t)$ \\
$\mathbf{v}_{2}=\frac{\partial}{\partial t}$ & $u^{(2)}=f(x, t-\epsilon)$ \\
& $\mathbf{v}_{3}=(u+1) \frac{\partial}{\partial u}$ & $u^{(3)}=e^{-\epsilon} f(x, t)+\epsilon$ \\
\hline $\mathbf{v}_{1}=\frac{\partial}{\partial x}$ & $u^{(1)}=f(x-\epsilon, t)$ \\
& $\mathbf{v}_{2}=\frac{\partial}{\partial t}$ & $u^{(2)}=f(x, t-\epsilon)$ \\
& $\mathbf{v}_{3}=2 t \frac{\partial}{\partial x}+\frac{\partial}{\partial u}$ & $u^{(3)}=f(x-2 \epsilon t, t)+\epsilon$ \\
& $\mathbf{v}_{4}=x \frac{\partial}{\partial x}+3 t \frac{\partial}{\partial t}-2 u \frac{\partial}{\partial u}$ & $u^{(4)}=e^{-2 \epsilon} f\left(e^{-\epsilon} x, e^{-3 \epsilon} t\right)$ \\
\hline
\end{tabular}

Integrating the last pair, we obtain that

$$
u=t^{-\alpha} v(\chi), \quad \alpha=\frac{2}{3(m-1)} .
$$

Now $u$ in (2.26) can be differentiated with respect to $t$ and $x$, where the derivatives of the symmetry variable are given by $\chi_{x}=t^{-1 / 3}, \chi_{t}=-t^{-4 / 3} x / 3$. We obtain

$$
u_{x}=t^{-\alpha-1 / 3} v^{\prime}, \quad u_{x x x}=t^{-\alpha-1} v^{\prime \prime \prime}, \quad u_{t}=t^{-\alpha-1}\left(-\alpha v-\frac{1}{3} \chi v^{\prime}\right)
$$

where differentiation here is with respect to $\chi$. Substituting these derivatives into (2.1), the equation takes the form

$$
v^{\prime \prime \prime}+m v^{m-1} v^{\prime}-\frac{1}{3} \chi v^{\prime}-\alpha v=0 .
$$

A similar analysis can be done with $\mathbf{v}_{4}$ in the third case, and it is found that the same form (2.28) is obtained with $\alpha=2 / 3$. In this case, the equation can be transformed into the form of a second Painlevé transcendent.

Now $\mathbf{v}_{1}$ and $\mathbf{v}_{2}$ are common in all three cases, and this suggests that a solution of the form

$$
u(x, t)=f(k x-\omega t)
$$


can be determined. In this case, the symmetry variable is $y=k x-\omega t$, and writing the required derivatives in terms of $y,(2.1)$ can be written as

$$
-\omega f_{y}+k^{3} f_{y y y}+k\left(f^{m}\right)_{y}=0 .
$$

Integrating this once, it reduces to a second-order equation,

$$
-\omega f+k^{3} f_{y y}+k f^{m}=\frac{1}{2} C_{0} .
$$

Multiplying on both sides by $f_{y}$ and integrating, we obtain the first-order equation for $f$,

$$
f_{y}^{2}=C_{0} f+\frac{\omega}{k^{3}} f^{2}-\frac{2}{k^{2}(m+1)} f^{m+1}+\gamma .
$$

This equation can be separated and then integrated on both sides to give

$$
\int \frac{d f}{\sqrt{C_{0} f+\left(\omega / k^{3}\right) f^{2}-\left(2 /(m+1) k^{2}\right) f^{m+1}+\gamma}}=\epsilon y+a, \quad \epsilon= \pm 1 .
$$

For specific values of $m$, large classes of solutions to (2.1) can be determined from (2.33) by varying $m$, in particular, elliptic function solutions.

As an example, we take $m=3$ and take $C_{0}=\gamma=0$ in (2.33) to obtain

$$
\int \frac{d f}{\left(f^{2}\left(A-B f^{2}\right)\right)^{1 / 2}}=\epsilon y+a
$$

where $A=\omega / k^{3}$ and $B=1 / 2 k^{2}$. Then $f$ is a solution of the following expression:

$$
\frac{f\left(A-B f^{2}\right)^{1 / 2}}{\left(f^{2}\left(A-B f^{2}\right)\right)^{1 / 2} \sqrt{A}} \ln \left(\frac{2\left(A+\sqrt{A}\left(A-B f^{2}\right)^{1 / 2}\right)}{f}\right)=\epsilon y-a .
$$

\section{Symmetries of the fully nonlinear equation}

This analysis can be extended to the case of (1.1) when $n \neq 1$. To apply $p r^{(3)} \mathbf{v}$ to (1.1), it should be expanded into its constituent derivatives in the following form:

$$
u_{t}+n(n-1)(n-2) u^{n-3} u_{x}^{3}+3 n(n-1) u^{n-2} u_{x} u_{x x}+n u^{n-1} u_{x x x}+m u^{m-1} u_{x}=0
$$

Applying the operator $p r^{(3)} \mathbf{v}$ to this differential equation, we obtain

$$
\begin{aligned}
\varphi^{t}+n(n-1)(n-2)(n-3) \varphi u^{n-4} u_{x}^{3}+3 n(n-1)(n-2) \varphi^{x} u^{n-3} u_{x}^{2} \\
+ \\
\quad+3 n(n-1)(n-2) \varphi u^{n-3} u_{x} u_{x x}+3 n(n-1) \varphi^{x} u^{n-2} u_{x x}+3 n(n-1) \varphi^{x x} u^{n-2} u_{x} \\
+n(n-1) \varphi u^{n-2} u_{x x x}+n \varphi^{x x x} u^{n-1}+m(m-1) \varphi u^{m-2} u_{x}+m \varphi^{x} u^{m-1}=0 .
\end{aligned}
$$

The next step is to substitute the coefficients of the prolongation operator into (3.2). It is understood that $u_{t}$ is replaced by (3.1), and the coefficients of the respective $x$ derivatives 
are collected and set to zero. Here, the expression obtained is much longer than that of the previous example, and most of the details will be left out. Starting with the highest derivatives $u_{x x t}, u_{x} u_{x x t}$ and $u_{x x}^{2}$, again we find that $\tau=\tau(t)$ and $\xi_{u}=0$. The coefficient of $u_{x x x}$ is then given by

$$
u^{n-1}\left(3 \xi_{x}-\tau_{t}\right)-(n-1) \varphi u^{n-2}=0 .
$$

Since $n \neq 1$, the only way to eliminate the term in $u^{n-2}$ is to require that $\varphi=0$. This immediately restricts the form of the symmetry generator. The coefficient of $u^{n-1}$ must also vanish, and this gives the constraint $\tau_{t}=3 \xi_{x}$, which can be integrated to yield $\xi(x, t)=$ $\left(\tau_{t} / 3\right) x+\sigma(t)$. The prolonged equation now collapses to the form

$$
\begin{aligned}
-\xi_{t} u_{x}+ & n(n-1)(n-2)\left(\tau_{t}-3 \xi_{x}\right) u^{n-3} u_{x}^{3}+3 n(n-1)\left(\tau_{t}-3 \xi_{x}\right) u^{n-2} u_{x} u_{x x} \\
& +m\left(\tau_{t}-\xi_{x}\right) u^{m-1} u_{x}=0 .
\end{aligned}
$$

The second and third terms vanish due to the constraint $\tau_{t}=3 \xi_{x}$. This requires that $\xi=c_{2}$ and $\tau=c_{1}$, where $c_{1}$ and $c_{2}$ are constants. Since $\varphi=0$, the general symmetry vector field is given by

$$
\mathbf{v}=c_{1} \frac{\partial}{\partial t}+c_{2} \frac{\partial}{\partial x}
$$

This is again a subalgebra, but even more restrictive than the previous case in which $n=1$. Only the two translational symmetries survive in this case. It is worth noting that the conclusions of this analysis are the same for the more general version of (1.1) given in the form

$$
u_{t}+\kappa\left(u^{m}\right)_{x}+\delta\left(u^{n}\right)_{x x x}=0
$$

where $\kappa$ and $\delta$ are real constants.

Based on the symmetry (3.5), group invariant solutions of the form

$$
u(x, t)=g(k x-\omega t)
$$

can be obtained. Using the symmetry variable $y=k x-\omega t$ and transforming the derivative into the $y$ variable, (1.1) can be written in the form

$$
-\omega g_{y}+k\left(g^{m}\right)_{y}+k^{3}\left(g^{n}\right)_{y y y}=0 .
$$

Integrating once, this takes the form

$$
n\left(g^{n-1} g_{y}\right)_{y}=\frac{\omega}{k^{3}} g-\frac{1}{k^{2}} g^{m}+C .
$$

Multiplying on both sides of this by $g^{n-1} g_{y}$, we can integrate both sides once more to find

$$
\left(g^{n-1} g_{y}\right)^{2}=\frac{2 \omega}{n(n+1) k^{3}} g^{n+1}-\frac{2}{n(m+n) k^{2}} g^{m+n}+\frac{2 C}{n^{2}} g^{n}+\gamma .
$$


Here, $C$ and $\gamma$ are constants of integration. Solving this for $g_{y}$, this equation can be separated and then integrated to give

$$
\int \frac{g^{n-1} d g}{\sqrt{2 \omega / n(n+1) k^{3} g^{n+1}-2 / n(m+n) k^{2} g^{m+n}+2 C / n^{2} g^{n}+\gamma}}=\epsilon y+a, \quad \epsilon= \pm 1 .
$$

The final integration constant is written $a$, which appears as a result of the last integration.

We work out the integral in (3.11) for several values of $m$ and $n$ in the case in which the constants of integration $C$ and $\gamma$ vanish. It may be assumed that $\omega$ and $k$ are positive constants.

Consider $n=m=2$ and set $\beta=4 \omega / 3 k$, then the integral may be written in the form

$$
2 k \int \frac{d g}{\sqrt{\beta g-g^{2}}}=\epsilon y+a, \quad \epsilon= \pm 1 .
$$

We take $\epsilon$ to be defined this way in what follows. This integral can be done, and solving for $g$, we obtain that

$$
g(y)=\frac{\beta}{2}\left(1+\sin \left(\frac{\epsilon y+a}{2 k}\right)\right)
$$

In the case in which $a=k \pi$, using the identity $1+\cos 2 x=2 \cos ^{2} x$, this takes the form of the compacton which has been discussed [11], namely,

$$
g(y)=\frac{4 \omega}{3 k} \cos ^{2}\left(\frac{y}{4 k}\right)
$$

This solution has the property that it is positive for $|y|<2 \pi k$ and zero at the endpoints. This fact enables us to define a compacton form of solution by taking a solution of the form

$$
u(x, t)= \begin{cases}\frac{4 \omega}{3 k} \cos ^{2}\left(\frac{k x-c t}{4 k}\right), & |k x-c t|<2 \pi k \\ 0, & |k x-c t|>2 \pi k\end{cases}
$$

Moreover, the derivative of this $u(x, t)$ has a derivative that is continuous at the endpoints of this interval.

Let $n=3, m=2$, then the integral reduces to

$$
\sqrt{\frac{15}{2}} k \int \frac{d g}{\sqrt{5 \omega / 4 k-g}}=\epsilon y+a
$$

It follows that $g(y)$ is given by

$$
g(y)=\frac{5 \omega}{4 k}-\frac{1}{30 k^{2}}(y+a)^{2} .
$$


2168 Symmetry properties of a generalized KdV equation

Let $n=2, m=3$, then with $\beta=5 \omega / 3 k$, the integral takes the form

$$
\sqrt{\frac{5}{2}} k \int \frac{d g}{\sqrt{\beta g-g^{3}}}=\epsilon y+a .
$$

Integrating and solving for $g$, a Jacobi elliptic function is obtained as a solution to (1.1) in this case,

$$
g(y)=\beta\left(\operatorname{sn}\left(\frac{\sqrt{-5 \sqrt{\beta}}(\epsilon y+a)}{2 k}, \frac{1}{\sqrt{2}}\right)^{2}-1\right) .
$$

Finally, for $n=3, m=3$, we set $\beta=3 \omega / 2 k$, then the integral takes the form

$$
3 k \int \frac{d g}{\sqrt{\beta-g^{2}}}=\epsilon y+a .
$$

Integrating and solving for $g(y)$, we have

$$
g(y)=\sqrt{\beta} \sin \left(\frac{\epsilon y+a}{3 k}\right) .
$$

Other cases could be integrated and would provide elliptic function solutions to (1.1).

\section{Reduction of equation subject to a differential constraint}

An interesting reduction of (2.1) takes place if we subject it to a differential constraint which has a structure analogous to that of a Schrödinger equation. To generate further solutions of (2.1), the following proposition can be used [2].

Proposition 4.1. Let $f(x, t)$ and $g(x, t)$ be functions which satisfy the differential equation

$$
\frac{\partial^{2} \psi}{\partial x^{2}}+\left[\frac{m}{2(m+1)} u^{m-1}+\lambda\right] \psi=0
$$

where $u$ is defined as

$$
u(x, t)=f(x, t) \cdot g(x, t),
$$

and $\lambda$ is a real constant. Then the generalized $K d V$ equation (2.1) reduces to the form of the first-order partial differential equation given as follows:

$$
f\left(\frac{\partial g}{\partial t}-4 \lambda \frac{\partial g}{\partial x}\right)+g\left(\frac{\partial f}{\partial t}-4 \lambda \frac{\partial f}{\partial x}\right)=0 .
$$

Proof. With $u$ defined by (4.2), we write the last nonlinear term in (2.1) in the form

$$
m u^{m-1} u_{x}=b(f g)^{m-1} \frac{\partial u}{\partial x}+a u^{m-1}\left(f \frac{\partial g}{\partial x}+g \frac{\partial f}{\partial x}\right),
$$


where $a$ and $b$ are constants which satisfy $a+b=m$. Differentiating $u$ with respect to $x$ and $t,(2.1)$ takes the form

$$
\begin{aligned}
f & \left\{\frac{\partial g}{\partial t}+\frac{\partial^{3} g}{\partial x^{3}}+\frac{3}{f} \frac{\partial^{2} f}{\partial x^{2}} \frac{\partial g}{\partial x}+a u^{m-1} \frac{\partial g}{\partial x}+\frac{b}{2} f^{m-2} g^{m-1} \frac{\partial u}{\partial x}\right\} \\
& +g\left\{\frac{\partial f}{\partial t}+\frac{\partial^{3} f}{\partial x^{3}}+\frac{3}{g} \frac{\partial^{2} g}{\partial x^{2}} \frac{\partial f}{\partial x}+a u^{m-1} \frac{\partial f}{\partial x}+\frac{b}{2} f^{m-1} g^{m-2} \frac{\partial u}{\partial x}\right\}=0 .
\end{aligned}
$$

Suppose that $f$ and $g$ are required to satisfy the equation

$$
\frac{\partial^{2} \psi}{\partial x^{2}}-\left[q u^{s}-\lambda\right] \psi=0
$$

where $u$ is given by (4.2). We show that we can pick $q$ and $s$ in a unique way such that the conclusion of the proposition holds. The third derivatives of $f$ and $g$ can be obtained by differentiating this constraint with respect to $x$. Substituting these derivatives into (4.5), the quantity inside the first bracket in (4.5) can be written in the form

$$
\frac{\partial g}{\partial t}+\left(q s f^{s-1} g^{s}+\frac{b}{2} f^{m-2} g^{m-1}\right) \frac{\partial u}{\partial x}+\left(4 q u^{s}+a u^{m-1}\right) \frac{\partial g}{\partial x}-4 \lambda \frac{\partial g}{\partial x} .
$$

If we take $s=m-1$, then the coefficients of the second and third terms reduce to the system of equations

$$
q(m-1)+\frac{b}{2}=0, \quad 4 q+a=0 .
$$

Solving these equations subject to the condition that $a+b=m$, we obtain the solution

$$
a=\frac{2 m}{m+1}, \quad b=\frac{m(m-1)}{m+1}, \quad q=-\frac{m}{2(m+1)} .
$$

This procedure can be repeated on the second bracket in (4.5) and exactly the same solution (4.9) for these constants is obtained. Therefore, combining the remaining terms in (4.5) then gives the result (4.3).

Now dividing both sides of (4.3) by (4.2), it is easy to see that, using the linearity of the derivative operators, (4.3) can be put in the equivalent form

$$
\left(\frac{\partial}{\partial t}-4 \lambda \frac{\partial}{\partial x}\right) \ln (f(x, t) \cdot g(x, t))=0 .
$$

This result implies that $u(x, t)$ has the particular structure

$$
u(x, t)=f(x, t) \cdot g(x, t)=h(x+4 \lambda t),
$$

where the function $h$ is unspecified for the moment.

We consider an instance in which $h$ can be determined explicitly by making use of the constraint (4.1) as an example. Consider the case in which

$$
f(x, t)=g(x, t)=\phi(x, t) .
$$


From the preceding considerations, $\phi(x, t)$ must have the form $\phi(x, t)=\phi(x+4 \lambda t)=$ $\phi(y)$, where $y=x+4 \lambda t$. Of course, $\phi(x, t)$ must satisfy the second-order (4.1) as well, which takes the form

$$
\ddot{\phi}+\frac{m}{2(m+1)} \phi^{2 m-1}+\lambda \phi=0 .
$$

Differentiation in (4.13) is with respect to the symmetry variable $y$. The constraint is sufficient to determine the function $h$ in this case. Multiplying (4.13) by $\dot{\phi}$, this can be integrated to give

$$
\dot{\phi}^{2}=C_{0}-\frac{1}{2(m+1)} \phi^{2 m}-\lambda \phi^{2}
$$

where $C_{0}$ is a constant of integration. This equation can finally be integrated by quadrature to give the integral

$$
\int \frac{d \phi}{\sqrt{C_{0}-(1 / 2(m+1)) \phi^{2 m}-\lambda \phi^{2}}}=y+a .
$$

The integral (4.15) will generate solutions to (2.1) in the form of elliptic functions. As an example, (4.15) can be integrated when $m=1$ to give a solution

$$
\phi(x, t)=2 \sqrt{\frac{C_{0}}{4 \lambda+1}} \sin \left(\frac{1}{2} \sqrt{4 \lambda+1}(x+4 \lambda t+a)\right) .
$$

Squaring (4.16), we obtain an explicit solution to (2.1) of the form $u(x, t)=\phi(x, t)^{2}$.

We now generalize a result in [2] to the case of (2.1).

Proposition 4.2. Suppose that $w=w(x, t)$ is a solution to the generalized KdV equation (2.1) and satisfies the additional constraint

$$
\frac{\partial^{2} w}{\partial x^{2}}-\frac{1}{w}\left(\frac{\partial w}{\partial x}\right)^{2}+\frac{m}{6} w\left(w^{m-1}-w^{-m+1}\right)=0 .
$$

Then the reciprocal $u=1 / w$ is a solution to (2.1).

To prove this, substitute $u(x, t)=1 / w(x, t)$ into (2.1). Replacing $w_{t}$ from (2.1) and $w_{x x}$ from (4.17), the result follows.

A first integral for (4.17) can be obtained of the form

$$
\left(\frac{\partial w}{\partial x}\right)^{2}=a w^{m+1}+b w^{-m+3}+K w^{2}
$$

where $a$ and $b$ will depend on $m$ and $K$ is a constant of integration. Differentiating both sides with respect to $x$, we obtain

$$
\frac{\partial^{2} w}{\partial x^{2}}=\frac{a}{2}(m+1) w^{m}-\frac{b}{2}(m-3) w^{-m+2}+K w .
$$


An expression for $K w$ can be obtained from (4.18), and substituting this into (4.19), there results

$$
\frac{\partial^{2} w}{\partial x^{2}}=\frac{1}{w}\left(\frac{\partial w}{\partial x}\right)^{2}+\left(\frac{m-1}{2}\right) a w^{m}-\left(\frac{m-1}{2}\right) b w^{-m+2}
$$

Comparing this to (4.17), it must be that $a=b=-m /(3(m-1))$ when $m \neq 1$. Thus, we have proved the following claim.

Proposition 4.3. When $m \neq 1$, the equation

$$
\left(\frac{\partial w}{\partial x}\right)^{2}=-\frac{m}{3(m-1)} w^{2}\left(w^{m-1}+w^{-m+1}\right)+K w^{2}
$$

is a first integral for (4.17).

More can be said with regard to the class of functions referred to in Proposition 4.2. This will generalize what was done in [2] for the usual KdV equation. Differentiating the first integral in (4.19) with respect to $x$, another relation for $w_{x x x}$ results in

$$
w_{x x x}=\frac{a}{2} m(m+1) w^{m-1} w_{x}+\frac{b}{2}(m-3)(m-2) w^{-m+1} w_{x}+K w_{x} .
$$

Replacing the third derivative (4.21) in (2.1), a first-order nonlinear equation is obtained:

$$
\left(\alpha w^{m-1}+\beta w^{-m+1}+K\right) w_{x}+w_{t}=0
$$

where

$$
\alpha=\frac{a}{2} m(m+1)+m, \quad \beta=\frac{b}{2}(m-3)(m-2) .
$$

Equation (4.23) is a quasilinear equation and the following initial value problem can be solved:

$$
\tau(w) w_{x}+w_{t}=0, \quad w(x, 0)=w_{0}(x)
$$

where $\tau(w)=\alpha w^{m-1}+\beta w^{-m+1}+K$. Consider the equivalent problem

$$
\begin{gathered}
x_{r}=\tau(w), \quad t_{r}=1, \quad w_{r}=0, \\
x(0, s)=s, \quad t(0, s)=0, \quad w(0, s)=w_{0}(s) .
\end{gathered}
$$

Here $w_{0}$ is an arbitrary function of one variable for the moment. Integrating this firstorder system, we obtain the result $w=w_{0}(s), t=r$, and $s=x-\tau(w) t$, from which it follows that

$$
w=w_{0}(x-\tau(w) t)
$$

This will actually furnish the solution to (4.26) provided that the equation $\Phi(x, t, w)=$ $w-w_{0}(x-\tau(w) t)=0$ can be solved for $w$ as a function of $x$ and $t$. Substituting (4.27) 
2172 Symmetry properties of a generalized KdV equation

into (4.18), it is reduced to an ordinary differential equation

$$
\dot{w}_{0}^{2}=a w_{0}^{m+1}+b w_{0}^{-m+3}+K w_{0}^{2}
$$

which can be put in the form of a quadrature

$$
\int \frac{d w_{0}}{\left(a w_{0}^{m+1}+b w_{0}^{-m+3}+K w_{0}^{2}\right)^{1 / 2}}= \pm(x-\tau(w) t)+c .
$$

This will determine the class of functions $w_{0}$ which will determine $w$ by solving (4.27) and satisfy Proposition 4.2.

The analog of Proposition 4.2 for (1.1) is as follows.

Proposition 4.4. Suppose that $w=w(x, t)$ is a solution of the generalized KdV equation (1.1) and in addition, satisfies the third-order constraint

$$
\begin{gathered}
\left(w^{n}-w^{-n+2}\right) \frac{\partial^{3} w}{\partial x^{3}}+\left[3\left((n+1) w^{-n+1}+(n-1) w^{n-1}\right) \frac{\partial^{2} w}{\partial x^{2}}+\frac{m}{n}\left(w^{m}-w^{-m+2}\right)\right] \frac{\partial w}{\partial x} \\
+\left(w^{n-2}\left(n^{2}-3 n+2\right)-w^{-n}\left(n^{2}+3 n+2\right)\right)\left(\frac{\partial w}{\partial x}\right)^{3}=0 .
\end{gathered}
$$

Then the reciprocal function $u=1 / w$ is also a solution of (1.1). When $n=1,(4.30)$ reduces to (4.17).

Proposition 4.5. When $m=n$ in (1.1), there exists a separation of variables solution of the form

$$
u(x, t)=f(x) \cdot g(t)
$$

provided that $f$ and $g$ can be found which satisfy the equations

$$
\begin{gathered}
\left(f^{n}\right)_{x}+\left(f^{n}\right)_{x x x}+\lambda f=0, \\
g_{t}-\lambda g^{n}=0 .
\end{gathered}
$$

The second equation in (4.32) can be integrated to give $g(t)$ from $(n-1) g^{n-1}=-(\lambda t+c)^{-1}$ when $n \neq 1$, and $g(t)=c e^{\lambda t}$ when $n=1$ and $c$ is a constant here.

\section{Summary}

To conclude, the symmetry group for the generalized KdV equation has been calculated. The translational symmetry which was found, although of frequent occurrence in such types of equations, for the case of compacton solutions leads to the idea of using this system to model sets of bubbles and droplets or bubble patterns. Thus, one can imagine sequences of bubbles which are juxtaposed in some order, such that the pattern can be translated into itself. In classical soliton theory, integrability and elastic collisions are closely connected. Some conservation laws have been found for (1.1) previously, but it is not known whether the equation is integrable [11]. It might be hoped that symmetry 
methods can be useful in searching for new conservation laws, and perhaps to help settle the question of integrability for this system.

\section{Acknowledgment}

I would like to thank Professor L. Debnath for suggesting the study of this equation to me.

\section{References}

[1] M. J. Ablowitz and H. Segur, Solitons and the Inverse Scattering Transform, SIAM Studies in Applied Mathematics, vol. 4, SIAM, Pennsylvania, 1981.

[2] P. Bracken, Some methods for generating solutions to the Korteweg-de Vries equation, Phys. A 335 (2004), no. 1-2, 70-78.

[3] A. Das, Integrable Models, World Scientific Lecture Notes in Physics, vol. 30, World Scientific, New Jersey, 1989.

[4] Y. S. Kivshar, Intrinsic localized modes as solitons with a compact support, Phys. Rev. E 48 (1993), no. 1, R43-R45.

[5] A. Ludu and J. P. Draayer, Patterns on liquid surfaces: cnoidal waves, compactons and scaling, Phys. D 123 (1998), no. 1-4, 82-91.

[6] R. M. Miura, The Korteweg-de Vries equation: a survey of results, SIAM Rev. 18 (1976), no. 3, $412-459$.

[7] A. C. Newell, The interrelation between Bäcklund transformations and the inverse scattering transform, Bäcklund Transformations, the Inverse Scattering Method, Solitons, and Their Applications (Workshop Contact Transformations, Vanderbilt Univ., Nashville, Tenn, 1974) (R. M. Miura, ed.), Lecture Notes in Math., vol. 515, Springer, Berlin, 1976, pp. 227-240.

[8] P. J. Olver, Symmetry and explicit solutions of partial differential equations, Appl. Numer. Math. 10 (1992), no. 3-4, 307-324.

[9] _ Applications of Lie Groups to Differential Equations, 2nd ed., Graduate Texts in Mathematics, vol. 107, Springer, New York, 1993.

[10] P. J. Olver and P. Rosenau, Group-invariant solutions of differential equations, SIAM J. Appl. Math. 47 (1987), no. 2, 263-278.

[11] P. Rosenau and J. Hyman, Compactons: Solitons with finite wavelength, Phys. Rev. Lett. 70 (1993), no. 5, 564-567.

[12] A. J. Sievers and S. Takeno, Intrinsic localized modes in anharmonic crystals, Phys. Rev. Lett. 61 (1988), 970-973.

Paul Bracken: Department of Mathematics, University of Texas-Pan American, Edinburg, TX 78539-2999, USA

E-mail address: bracken@panam.edu 


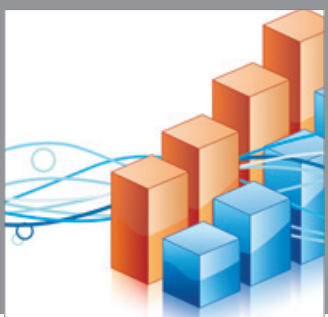

Advances in

Operations Research

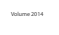

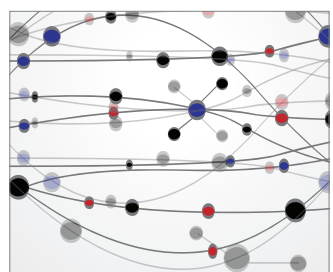

\section{The Scientific} World Journal
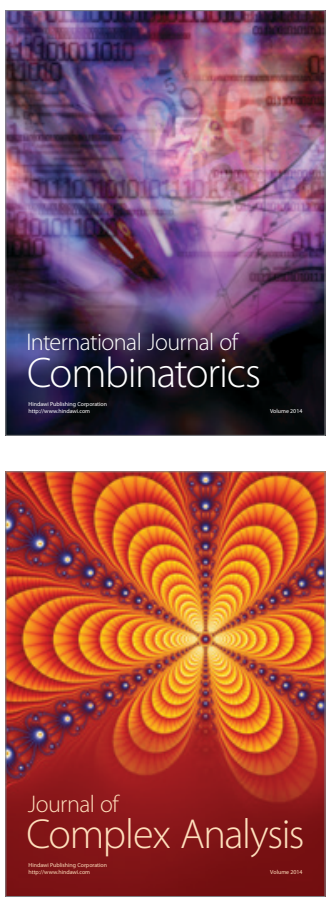

International Journal of

Mathematics and

Mathematical

Sciences
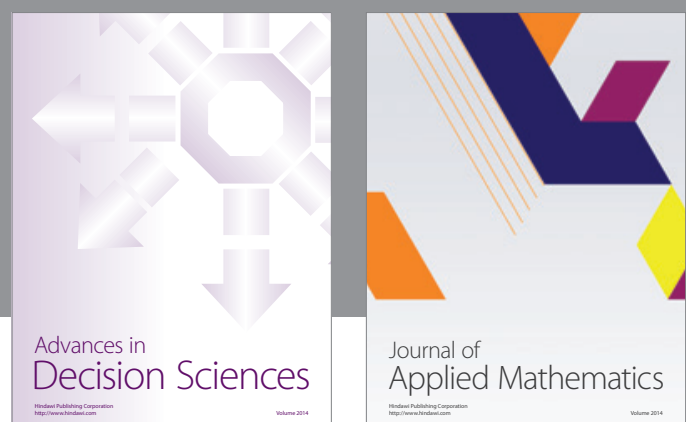

Journal of

Applied Mathematics
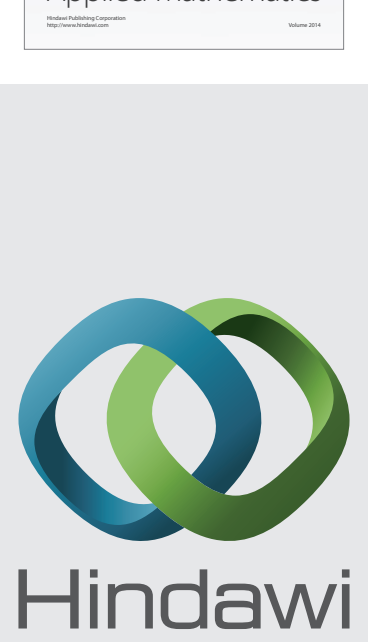

Submit your manuscripts at http://www.hindawi.com
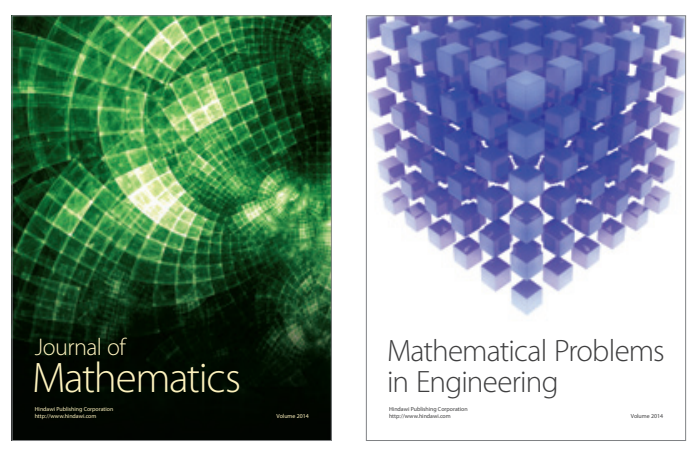

Mathematical Problems in Engineering
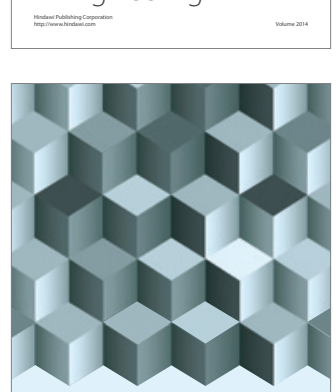

Journal of

Function Spaces
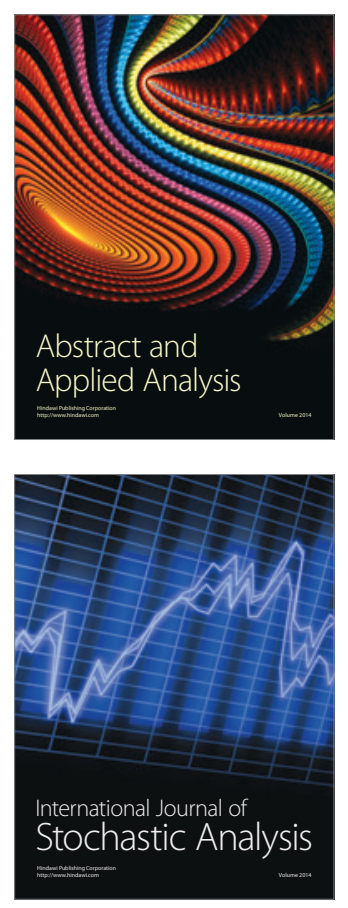

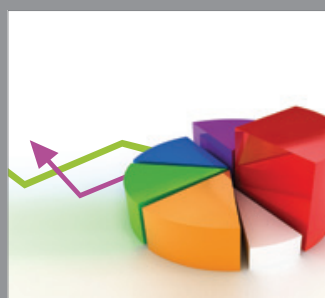

ournal of

Probability and Statistics

Promensencen
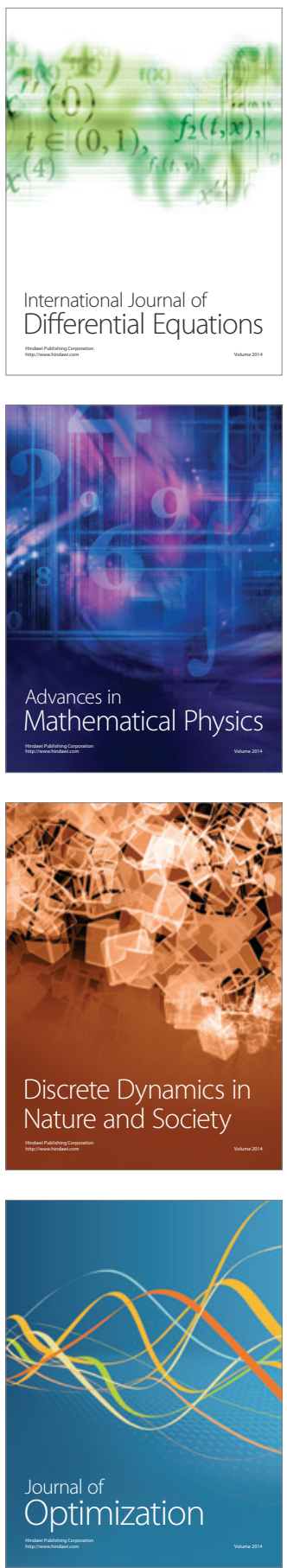